CHRONIC OBSTRUCTIVE PULMONARY DISEASE

\title{
Inflammatory markers are associated with ventilatory limitation and muscle dysfunction in obstructive lung disease in well functioning elderly subjects
}

\author{
S Yende, G W Waterer, E A Tolley, A B Newman, D C Bauer, D R Taaffe, R Jensen, \\ R Crapo, S Rubin, M Nevitt, E M Simonsick, S Satterfield, T Harris, S B Kritchevsky
}

See end of article for authors' affiliations

Correspondence to: Dr S Yende, Department of Critical Care Medicine, University of Pittsburgh, Pittsburgh, PA 15238, USA; yendes@upmc.edu

Received 2 September 2004

Accepted 18 October 2005

Published Online First

11 November 2005

Thorax 2006;61:10-16. doi: 10.1136/thx.2004.034181

Background: Inflammatory markers are increased in chronic obstructive pulmonary disease (COPD) and are hypothesised to play an important part in muscle dysfunction and exercise intolerance.

Methods: The Health Aging and Body Composition (Health $A B C$ ) study is a prospective observational cohort of well functioning individuals aged 70-79 years. A cross sectional analysis of the baseline data was conducted to examine the association between inflammatory markers and ventilatory limitation, muscle strength, and exercise capacity. These associations were compared in participants with and without obstructive lung disease (OLD).

Results: Of the 3075 participants enrolled in the Health ABC cohort, OLD was identified by spirometric testing in 268 participants and 2005 participants had normal spirometric results. Of the participants with OLD, $35 \%, 38 \%$, and $27 \%$ participants had mild, moderate, and severe OLD, respectively. Participants with OLD had lower quadriceps strength ( $102.5 \mathrm{Nm} v 108.9 \mathrm{Nm}, \mathrm{p}=0.02$ ), lower maximum inspiratory pressure $\left(64.7 \mathrm{~cm} \mathrm{H} \mathrm{H}_{2} \mathrm{O} v 74.2 \mathrm{~cm} \mathrm{H} \mathrm{H}_{2} \mathrm{O}, \mathrm{p}<0.0001\right)$, higher systemic interleukin (IL)- 6 levels $(2.6 \mathrm{pg} / \mathrm{ml} v$ $2.2 \mathrm{pg} / \mathrm{ml}, \mathrm{p}<0.0001$ ), and higher C-reactive protein (CRP) levels ( $3.5 \mathrm{mg} / \mathrm{l} \vee 2.5 \mathrm{mg} / \mathrm{l}, \mathrm{p}<0.0001$ ) than those with normal spirometry. In participants with OLD and those with normal spirometry, forced expiratory volume in 1 second $\left(F E V_{1}\right)$ was associated with IL- 6 (adjusted regression coefficients $(\beta)=-5.3$ $(95 \% \mathrm{Cl}-9.1$ to -1.5$)$ and $-3.1(95 \% \mathrm{Cl}-4.3$ to -1.9$)$, respectively). IL-6 and TNF were also associated with quadriceps strength among participants with OLD and those with normal spirometry ( $\beta=-6.4$ (95\% $\mathrm{Cl}-12.8$ to -0.03$)$ and $-3.4(95 \% \mathrm{Cl}-5.4$ to -1.3$)$, respectively, for IL-6 and $\beta=-10.1(95 \% \mathrm{Cl}$ -18.7 to -1.5$)$ and $-3.8(95 \% \mathrm{Cl}-7$ to -0.6$)$, respectively, for TNF). IL-6, quadriceps strength, and maximum inspiratory pressures were independent predictors of reduced exercise capacity in both groups. Conclusions: In well functioning elderly subjects with or without OLD, IL-6 is associated with reduced FEV quadriceps strength, and exercise capacity.

C hronic obstructive pulmonary disease (COPD) is associated with systemic inflammation, ${ }^{1}$ which is increas-

ingly recognised as a risk factor for its systemic complications such as muscle dysfunction, coronary artery disease, $^{2-5}$ and stroke. ${ }^{6}$ Skeletal muscle dysfunction, with preferential involvement of quadriceps muscles, is an important cause of reduced exercise capacity in patients with COPD. ${ }^{7-10}$ Cachexia rather than nutritional imbalance causes skeletal muscle wasting in COPD. ${ }^{8}$

Numerous ex vivo studies have shown that systemic inflammatory markers such as tumour necrosis factor $\alpha$ $(\mathrm{TNF}-\alpha)$ and interleukin (IL)-6 are proximal markers of cachexia and selectively target myosin in skeletal muscles. ${ }^{8}{ }^{11-13}$ While TNF reduces synthesis of myosin, IL-6 increases degradation of myosin through the ubiquitin-proteasome pathway. Furthermore, these cytokines may cause weakness without muscle wasting by directly compromising contractile function. ${ }^{14}$ Despite increasing evidence for the role of cytokines in skeletal muscle wasting in COPD patients, few studies have confirmed this association in humans. ${ }^{15-18} \mathrm{~A}$ recent study characterised the relationship between IL-6 and skeletal muscle strength during exacerbations of COPD but failed to show an association during stable COPD. ${ }^{19}$ Using creatinine-height index to assess skeletal muscle mass, Eid et al found an association between body composition and skeletal muscle mass with systemic inflammatory markers in COPD. ${ }^{20}$ However, the relationship between inflammatory markers and strength in specific groups of muscles in individuals with stable COPD remains unclear.

The Health Aging and Body Composition (Health ABC) study is a prospective observational cohort of 70-79 year old well functioning elderly subjects with spirometric data and levels of inflammatory markers including TNF, IL-6, and Creactive protein (CRP) at baseline in most participants. A cross sectional analysis of these baseline data was undertaken to ascertain the relationship between lung function and inflammatory markers, and between inflammatory markers and muscle strength, in study participants with obstructive lung disease (OLD) and those with normal spirometric parameters. Predictors of exercise intolerance in participants with OLD and those with normal spirometric data were also estimated.

\section{METHODS}

Study population

The Health ABC study cohort consists of 3075 men and women aged $70-79$ years. This study was designed to assess prospectively the impact of health conditions and body composition on incident mobility limitation. Inclusion criteria included no reported difficulty with walking a quarter

Abbreviations: $\mathrm{CRP}, \mathrm{C}$-reactive protein; $\mathrm{FEV}_{1}$, forced expiratory volume in 1 second; IL, interleukin; OLD, obstructive lung disease; PImax, maximum inspiratory pressure; TNF, tumour necrosis factor 
of a mile, climbing 10 steps, or performing activities of daily living. Exclusion criteria included treatment for cancer, enrolment in a trial of a lifestyle intervention, plans to move out of the study area in 3 years, difficulty in communicating with study personnel, and cognitive impairment. This analysis is restricted to 268 participants with obstructive pattern and 2005 with normal spirometric parameters. Reasons for excluding the remaining participants are shown in fig 1 .

\section{Demographic data, health conditions, and body composition}

Age, sex, race, and prevalent health conditions were assessed based on self report and medication inventory. Height was measured using a wall mounted stadiometer and body mass index (BMI) was calculated as weight $(\mathrm{kg}) /$ height $^{2}\left(\mathrm{~m}^{2}\right)$. The cross sectional areas of muscle, subcutaneous and intermuscular fat, and muscle density in both thighs were measured by CT scanning (Memphis clinic site: Somatom Plus 4, Siemens, Erlangen, Germany or PQ 2000S, Marconi Medical Systems, Cleveland, OH, USA; Pittsburgh clinic site: 9800 Advantage, General Electric, Milwaukee, WI, USA) as described previously. ${ }^{21}$ Whole body and lower limb lean mass was assessed by dual energy $x$ ray absorptiometry (DXA; Hologic 4500A, Waltham, MA, USA). Mean values for right and left thigh muscle area and lower limb lean body mass were used in the analysis.

\section{Muscle strength}

The maximal and mean isokinetic strength of the knee extensors (Nm) was assessed by a Kin-Com 125 AP Dynamometer (Chattanooga, TN, USA) at $60 \%$ and calculated from the average of three reproducible and acceptable trials out of a maximum of six trials. Handgrip strength was measured using a Jamar Hydrolic Hand Dynamometer.

\section{Spirometric tests}

Spirometric tests were performed approximately 2 weeks after the baseline interview using a horizontal dry rolling seal spirometer (SensorMedics Corporation, Yorba Linda, CA, USA). The spirometers were modified at the National Institute of Occupational Safety and Health (Morgantown,

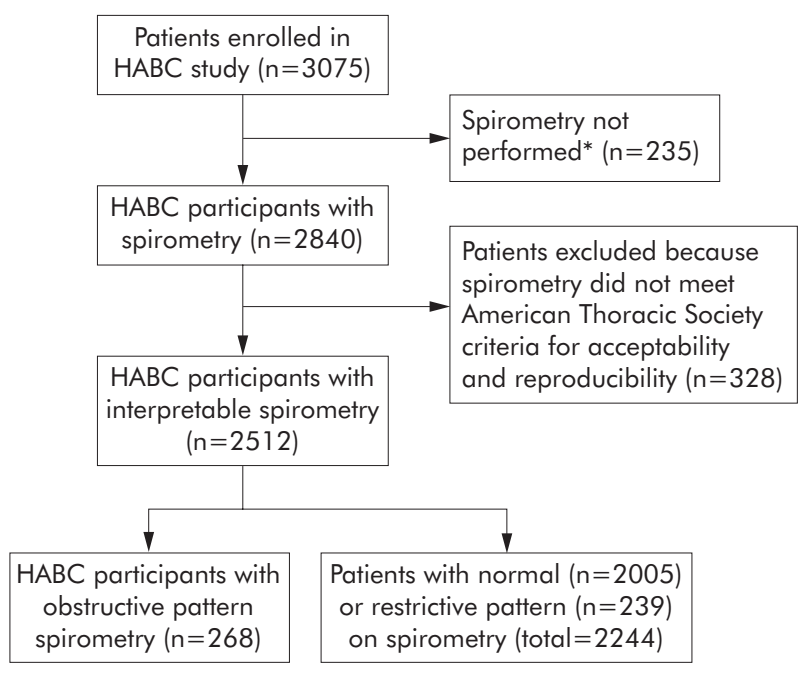

Figure 1 Reasons for excluding participants in the Health $A B C$ (HABC) study. *Reasons for not performing spirometry were medical. contraindications ( $n=160$ ), participant unable to follow test instructions $(n=12)$ or to complete the test $(n=15)$, participant refusal $(n=27)$, equipment failure $(n=1)$, and unknown $(n=20)$.
West Virginia) under the direction of Dr John Hankinson by adding an optical shaft encoder to measure piston displacement and installing the spirometry software used in the third National Health and Nutrition Examination survey. ${ }^{22}$ Contraindications to spirometric testing were recent abdominal, thoracic or ophthalmological surgery, recent myocardial infarction, or other recent cardiac illness.

A minimum of three and a maximum of five tracings were obtained from each subject. To be acceptable, tracings had to reflect a good start of test with no coughs or hesitations within the first second and a reasonable duration of test (a minimum of 6 seconds of exhalation or an acceptable plateau in the volume-time tracing). Forced vital capacity (FVC) and forced expiratory volume in 1 second $\left(\mathrm{FEV}_{1}\right)$ had to meet American Thoracic Society (ATS) criteria for acceptability and reproducibility. ${ }^{23}{ }^{24}$ OLD was defined as a reduced $\mathrm{FEV}_{\mathrm{l}} / \mathrm{FVC}$ as determined by age, sex, and race normalised values. ${ }^{22}$ Based on percentage predicted $\mathrm{FEV}_{1}$ determined by age, sex, height, and race, participants with OLD were divided into mutually exclusive groups: severe $\left(\mathrm{FEV}_{1} \leqslant 50 \%\right.$ predicted), moderate $\left(\mathrm{FEV}_{1} 51-70 \%\right.$ predicted), and mild $\left(\mathrm{FEV}_{1} \geqslant 70 \%\right.$ predicted). Maximum inspiratory pressure (PImax) was measured using an MRP-1 instrument (S\&M Instruments Inc).

\section{Inflammatory markers}

Blood samples were obtained in the morning (mean time 09.25 hours) at the year 1 visit. After processing the specimens were aliquoted into cryovials, frozen at $-70^{\circ} \mathrm{C}$, and shipped to the Core Laboratory. Citrated plasma was used for analysis of TNF and IL-6 assays and special coagulation (SCAT-I) plasma for CRP. ${ }^{25}$ Plasma TNF and IL-6 levels were measured in duplicate by ELISA kits (R\&D Systems, Minneapolis, MN, USA) using HS600 Quantikine and HSTA50 kits, respectively. Plasma levels of CRP were also measured in duplicate by ELISA based on purified protein and polyclonal anti-CRP antibodies (Calbiochem, EMD Biosciences Inc, Darmstadt, Germany). The CRP assay was standardised according to the World Health Organization First International Reference Standard. Reliability was ascertained using a blind duplicate system and interassay coefficients of variation for TNF, IL-6, and CRP assays were $16 \%, 10 \%$, and $8 \%$, respectively. The minimal detectable limits for TNF, IL-6, and CRP were $0.18 \mathrm{pg} / \mathrm{ml}, 0.1 \mathrm{pg} / \mathrm{ml}$ and $0.007 \mathrm{mg} / \mathrm{l}$, respectively. TNF, IL-6, and CRP data were missing in $6.6 \%, 5.3 \%$, and $2.3 \%$ of participants, respectively.

\section{Long distance corridor walk}

Exercise tolerance was measured by the long distance corridor walk test (LDCW) which covered 400 metres. The LDCW test differs from the 6 minute walk test because it uses a target distance instead of time. The LDCW test was therefore chosen to estimate exercise capacity because it discriminates walking endurance and cardiorespiratory fitness better than the 6 minute walk test for this age group. ${ }^{26}$ Participants were asked to walk 400 metres after a 2 minute warm-up. The course consisted of 10 laps in a long hallway around cones set 20 metres apart (40 metres per lap) with instructions to walk "as quickly as you can at a pace that you can maintain for the full 10 laps", with standard encouragement. Persons with baseline electrocardiogram (ECG) abnormalities, raised blood pressure ( $\geqslant 200 / 110 \mathrm{~mm} \mathrm{Hg})$, resting heart rate $>120$ or $<40$ beats per minute (bpm), recent exacerbation of chest pain, shortness of breath, or reporting a recent cardiac event or procedure were excluded for safety reasons. The test was stopped if heart rate exceeded $135 \mathrm{bpm}$ or if a participant reported chest pain or dyspnoea during the test. 


\begin{tabular}{|c|c|c|c|}
\hline Variable & $\begin{array}{l}\text { With OLD } \\
(n=268)\end{array}$ & $\begin{array}{l}\text { Without OLD } \\
(\mathbf{n}=2005)\end{array}$ & p value \\
\hline \multicolumn{4}{|l|}{ Demographic characteristics } \\
\hline Age & $73.6(2.8)$ & $73.2(2.8)$ & 0.03 \\
\hline Sex (\% male) & 58 & 50 & 0.02 \\
\hline Race (\% black) & 56 & 62 & 0.03 \\
\hline Site (\% Memphis) & 51 & 49 & 0.4 \\
\hline \multicolumn{4}{|l|}{ Co-morbid conditions } \\
\hline Hypertension (\%) & 47 & 50 & 0.3 \\
\hline Diabetes (\%) & 14 & 15 & 0.4 \\
\hline History of CHF (\%) & 0.8 & 1.2 & 0.8 \\
\hline History of COPD (\%) & 41.8 & 10.6 & $<0.0001$ \\
\hline Current smoking (\%) & 32 & 9 & 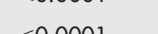 \\
\hline Past smoking (\%) & 54 & 46 & $<0.0001$ \\
\hline Pack years of smoking $\ddagger$ & $39(9-57)$ & $2(0-25)$ & $<0.0001$ \\
\hline Depression (\%) & 11.4 & 12.5 & \\
\hline \multicolumn{4}{|l|}{ Pulmonary function } \\
\hline $\mathrm{FEV}_{1}(\%$ predicted $)$ & $65.1(16.6)$ & $98.6(18.2)$ & $<0.0001$ \\
\hline Mild $\geqslant 70 \%$ predicted (\%) & & 100 & \\
\hline Moderate $51-70 \%$ predicted (\%) & 38 & 0 & \\
\hline Severe $<50 \%$ predicted $(\%)$ & 27 & 0 & \\
\hline \multicolumn{4}{|l|}{ Body composition } \\
\hline Body mass index $\left(\mathrm{kg} / \mathrm{m}^{2}\right)$ & $25.4(4.7)$ & $27.4(4.7)$ & 0.001 \\
\hline Visceral adiposity $\left(\mathrm{cm}^{2}\right)$ & 149.4 (77) & $146.9(67)$ & 0.4 \\
\hline Lean body mass $(\mathrm{kg})$ & $47(10)$ & $48(10)$ & 0.008 \\
\hline Weight $(\mathrm{kg})$ & $72(14)$ & $76(15)$ & $<0.0001$ \\
\hline \multicolumn{4}{|l|}{ Muscle strength and muscle mass } \\
\hline Grip strength $(\mathrm{kg})$ & $34.1(8)$ & $32.5(7)$ & 0.3 \\
\hline Knee extensor strength $(\mathrm{Nm})$ & $102.5(39)$ & $108.9(38)$ & 0.017 \\
\hline Thigh muscle area by CT scan $\left(\mathrm{cm}^{2}\right)$ & $102(39)$ & $108(39)$ & 0.002 \\
\hline Lower limb lean mass by DXA (kg) & $7.4(1.7)$ & $7.6(1.8)$ & 0.04 \\
\hline $\begin{array}{l}\text { Knee extensor strength/thigh muscle area by } \\
\text { CT scan }\left(\mathrm{Nm} / \mathrm{cm}^{2}\right)\end{array}$ & $0.95(0.24)$ & $0.95(0.22)$ & 0.9 \\
\hline Maximal inspiratory pressure $\left(\mathrm{cm} \mathrm{H}_{2} \mathrm{O}\right)$ & $64.7(30)$ & $74.2(32)$ & $<0.0001$ \\
\hline \multicolumn{4}{|l|}{ Inflammatory markerst } \\
\hline TNF (pg/ml) & $3.4(1.7)$ & $3.4(1.5)$ & 0.3 \\
\hline IL-6 (pg/ml) & 2.6 (1.9) & $2.2(1.8)$ & $<0.0001$ \\
\hline CRP (mg/l) & 3.5 (5.3) & $2.5(3.7)$ & $<0.0001$ \\
\hline \multicolumn{4}{|l|}{ Medications } \\
\hline Steroids (\%) & 4.5 & 1.2 & 0.006 \\
\hline $\begin{array}{l}\text { CHF, congestive heart failure; } F E V_{1} \text {, forced expir } \\
\text { TNF, tumour necrosis factor. } \\
\text { Data expressed as mean (SD) unless specified o } \\
\text { tData log converted since distribution not norm } \\
\text { †Median with interquartile range. }\end{array}$ & $\begin{array}{l}\text { volume in } 1 \text { se } \\
\text { ise. }\end{array}$ & RP, C-reactive & ein; IL, interleukir \\
\hline
\end{tabular}

\section{Statistical analysis}

Baseline characteristics in participants with and without OLD were compared with the $\chi^{2}$ test (discrete variables) or Student's $t$ test (continuous variables). A p value of $<0.05$ was considered significant. Inflammatory markers were log converted because distribution was not normal. Multiple linear regression analysis was used to estimate adjusted regression coefficients between each inflammatory marker and percentage predicted $\mathrm{FEV}_{1}$ as well as muscle strength. Age, sex, race, and the sex-race interaction if significant, site, BMI $>30,20-30$, and $<20 \mathrm{~kg} / \mathrm{m}^{2}$, smoking status (current smokers $v$ previous smokers $v$ never smokers), pack years of smoking, history of congestive heart failure, diabetes, and use of oral steroids were added to this model to control for confounding. These variables were chosen based on known associations with inflammatory markers, lung function, or muscle strength in our cohort or previous studies.

We also performed multivariable analysis using stepwise logistic regression to ascertain predictors of exercise capacity among participants with OLD. Age, sex, race, site, percentage predicted $\mathrm{FEV}_{1}$, history of congestive heart failure, quadriceps strength, maximum inspiratory strength, and inflammatory markers were entered into the model. As described previously, exercise tolerance was measured based on time to complete the LDCW test. Participants were stratified into three groups: those who did not participate in the LDCW test, individuals requiring $>6$ minutes to complete the LDCW test, and those requiring $<6$ minutes to complete the LDCW test. The 6 minute time point was chosen to compare distance covered in this test with a 6 minute walk test. A number of the participants were unable to perform the LDCW test. A multivariable analysis to examine predictors of exercise tolerance without these participants could potentially bias the estimates. We therefore compared odds ratios in individuals who required $<6$ minutes to complete the LDCW test with those who could not participate in the test or required $>6$ minutes to complete it. Odds ratios and $95 \%$ confidence intervals were estimated for participants with OLD and compared with those with normal spirometric data. Model fit was estimated by Hosmer-Lemeshow's test.

To ascertain if these associations were significantly different between participants with and without OLD, the results were stratified by these two groups and interaction $p$ values were estimated in each of these models. All analyses were performed using SAS Version 9.0 (Cary, NC, USA).

\section{RESULTS}

\section{Baseline characteristics}

Table I compares the baseline characteristics of the 268 participants with OLD and those with normal spirometric 
Table 2 Adjusted regression coefficients (with 95\% confidence intervals) for association between inflammatory markers and $\mathrm{FEV}_{1} \%$ predicted in participants with and without obstructive lung disease (OLD)

\begin{tabular}{llll}
\hline & \multicolumn{2}{l}{$\beta$ with 95\% confidence intervals* } & p value \\
\cline { 2 - 4 } Variable & With OLD & Without OLD & 0.09 \\
\hline IL-6 & $-5.3(-9.1$ to -1.5$)$ & $-3.1(-4.3$ to -1.9$)$ & 0.03 \\
TNF & $3.6(-1.8$ to 9$)$ & $-1.9(-3.7$ to 0.1$)$ & 0.6 \\
CRP & $-2.2(-4.8$ to 0.4$)$ & $-2(-3$ to -1$)$ & \\
\hline
\end{tabular}

IL-6, interleukin 6; TNF, tumour necrosis factor; CRP, C-reactive protein.

*Regression coefficients $(\beta)$ denote the estimated difference in $\mathrm{FEV}_{1} \%$ predicted associated with an increase of one log unit in individual inflammatory markers. $\beta$ adjusted for age, race, sex, site, smoking status (current smokers, ever smoked versus never smoked), pack years of smoking, body mass index ( $>30$ and $\left.<20 \mathrm{~kg} / \mathrm{m}^{2}\right)$, history of congestive heart failure, diabetes, and oral steroid use.

$\mathrm{tp}$ value for interaction between obstructive lung disease and the association between $\mathrm{FEV}_{1}$ and inflammatory marker.

parameters. Among the participants with OLD, 94 (35\%), 102 $(38 \%)$, and $72(27 \%)$ participants had mild $\left(\mathrm{FEV}_{1} \geqslant 70 \%\right.$ predicted), moderate $\left(\mathrm{FEV}_{1} 51-70 \%\right.$ predicted $)$, and severe $\left(\mathrm{FEV}_{1}<50 \%\right.$ predicted) OLD, respectively. Participants with OLD were more likely to be men, of black ethnicity, using oral steroid medications, and to be current as well as past smokers. The association between age and OLD was statistically significant but the differences were not clinically meaningful. Site of enrolment and history of hypertension, diabetes, congestive heart failure, or depression were not associated with OLD. Only $42 \%$ of participants with OLD reported a history of COPD.

Participants with OLD had lower BMI, total body weight, and lean body mass than those without OLD. Similarly, knee extensor strength, thigh muscle cross sectional area by CT scan, and maximum inspiratory pressure were lower in participants with OLD, although grip strength was similar in the two groups. Despite the lower knee extensor strength and thigh muscle cross sectional area by CT scan among the participants with OLD, the ratios of knee extensor strength and thigh muscle area were similar for both groups.

Table 3 Adjusted regression coefficients (with 95\% confidence intervals) for association between inflammatory markers (log converted) and quadriceps strength, grip strength, and maximum inspiratory pressure (PImax) in participants with and without obstructive lung disease (OLD)

\begin{tabular}{lll}
\hline & \multicolumn{2}{l}{$\beta$ with 95\% confidence intervals* } \\
\cline { 2 - 3 } Variable & With OLD & Without OLD \\
\hline Quadriceps strength & $-6.4(-12.8$ to -0.03$)$ & $-3.4(-5.4$ to -1.3$)$ \\
IL-6 & $-10.1(-18.7$ to -1.5$)$ & $-3.8(-7$ to -0.6$)$ \\
TNF & $-1.2(-5.4$ to 3.1$)$ & $-0.9(-2.4$ to 0.7$)$ \\
CRP & $-1.2(-2.8$ to 0.3$)$ & $-0.8(-1.3$ to -0.3$)$ \\
Grip strength & $-0.2(-2.2$ to 1.8$)$ & $-0.6(-1.4$ to 0.1$)$ \\
IL-6 & $0.1(-0.9$ to 1.1$)$ & $-0.4(-0.8$ to -0.02$)$ \\
TNF & $-4.2(-10.3$ to 1.8$)$ & $-0.8(-3.1$ to 1.5$)$ \\
CRP & $-6(-14.7$ to 2.7$)$ & $-3.5(-7.1$ to -0.003$)$ \\
PImax & $-1.2(-5.3$ to 2.9$)$ & $-0.02(-1.7$ to 1.8$)$ \\
IL-6 &
\end{tabular}

${ }^{*} \beta$ adjusted for age, race, sex, site, lean body mass, total fat, $\mathrm{FEV}_{1} \%$ predicted, smoking status (current smokers, ever smoked versus never smoked), pack years of smoking, body mass index ( $>30$ and $<20 \mathrm{~kg} /$ $\mathrm{m}^{2}$ ), history of diabetes and congestive heart failure, and use of oral steroids interaction. $p$ values for interaction between OLD and the association between individual inflammatory markers and muscle strength $>0.5$.

\section{Association between lung function and muscle strength}

The unadjusted mean values of quadriceps strength for participants with mild, moderate, and severe OLD were 104.6 Nm, 102.9 Nm, and 98.7 Nm, respectively, compared with $108.9 \mathrm{Nm}$ for those with normal spirometry. This negative association between $\mathrm{FEV}_{1} \%$ predicted and quadriceps strength was statistically significant $(p=0.007)$ after adjusting for age, height, sex, race, and sex-race interaction. In contrast to quadriceps strength, grip strength was similar in participants with mild, moderate, and severe OLD, and no relationship was noted with $\mathrm{FEV}_{1} \%$ predicted. PImax for those with mild, moderate, and severe OLD was $66.9 \mathrm{~cm} \mathrm{H}_{2} \mathrm{O}, 65.3 \mathrm{~cm} \mathrm{H}_{2} \mathrm{O}$, and $60 \mathrm{~cm} \mathrm{H}_{2} \mathrm{O}$, respectively, compared with $74.2 \mathrm{~cm} \mathrm{H}_{2} \mathrm{O}$ for those with normal spirometry. Similar to quadriceps strength, Pimax also correlated with $\mathrm{FEV}_{1} \%$ predicted $(\mathrm{p}=0.03)$ after adjusting for sex and race. Although weight, BMI, and lean body mass were lower in participants with OLD, none of these variables correlated with $\mathrm{FEV}_{1} \%$ predicted $(\mathrm{p}>0.05)$.

\section{Association between inflammatory markers and lung function}

TNF levels were similar but IL-6 and CRP levels were higher in participants with OLD than in those with normal spirometry. A modest correlation was noted between IL-6 levels and TNF and CRP (Spearman correlation coefficients $(r)=0.3$ and 0.5 , respectively, $\mathrm{p}<0.0001)$. However, only a weak correlation was noted between TNF and CRP levels $(r=0.1, \mathrm{p}=0.07)$.

IL-6 was associated with $\mathrm{FEV}_{1} \%$ predicted in participants with OLD and in those with normal spirometry (table 2). Although the adjusted regression coefficients were different for these two groups $(\beta=-5.3$ and -3.1 , respectively), the $p$ value for the interaction did not meet statistical significance $(p=0.09)$. In contrast, TNF was not associated with $\mathrm{FEV}_{1} \%$ predicted in either group. Although the adjusted regression coefficients for the association between CRP and $\mathrm{FEV}_{1} \%$ predicted were similar in participants with OLD and those with normal spirometry, the association was significant only in participants with normal spirometry.

\section{Association between inflammatory markers and muscle strength}

Both TNF and IL-6 were associated with quadriceps strength in participants with OLD and in those with normal spirometry (table 3 ). Although the adjusted regression coefficients were different for the two groups for both TNF and IL-6, the interaction $\mathrm{p}$ values were not significant $(\mathrm{p}=0.3$ and 0.6 , respectively). CRP was not associated with 


\begin{tabular}{|c|c|c|c|}
\hline Variable & More than $6 \mathrm{~min}$ & Did not participate & $\begin{array}{l}\text { Completed test } \\
\text { within } 6 \mathrm{~min}\end{array}$ \\
\hline \multicolumn{4}{|l|}{ With obstructive lung disease } \\
\hline Knee extensor strength $(\mathrm{Nm}) \dagger$ & $0.4(0.2$ to 0.7$)$ & $0.6(0.4$ to 0.8$)$ & Referent \\
\hline IL-6 (log value)† & 2.2 (1.1 to 4.4$)$ & $1.6(1.1$ to 2.5$)$ & Referent \\
\hline PImaxt & $0.5(0.3$ to 0.8$)$ & 0.7 (0.5 to 0.9$)$ & Referent \\
\hline \multicolumn{4}{|l|}{ Without obstructive lung disease } \\
\hline Knee extensor strength $(\mathrm{Nm}) \dagger$ & $0.5(0.4$ to 0.7$)$ & $0.7(0.6$ to 0.8$)$ & Referent \\
\hline IL-6 (log value)t & $1.9(1.5$ to 2.4$)$ & $1.6(1.4$ to 1.9$)$ & Referent \\
\hline PImax $\dagger$ & $0.8(0.7$ to 0.9$)$ & $0.9(0.8$ to 0.98$)$ & Referent \\
\hline
\end{tabular}

quadriceps strength in either group. None of the inflammatory mediators was associated with hand grip strength or Pimax, except for a weak association between IL-6 and hand grip strength, and between TNF and PImax in participants with normal spirometry.

\section{Predictors of exercise tolerance}

$30 \%$ of study participants with OLD did not participate in the LDCW. The quadriceps strength for those who did not participate in the LDCW or who took $>6$ minutes to complete the LDCW was $91 \mathrm{Nm}$ and $92 \mathrm{Nm}$, respectively, which was lower than the mean quadriceps strength for individuals who completed the LDCW test within 6 minutes (114 Nm). Similarly, IL-6 levels were higher in these two groups (mean $3.3 \mathrm{ng} / \mathrm{ml}$ for both groups) than in those who completed the test within 6 minutes (mean $2.3 \mathrm{ng} / \mathrm{ml}$ ). We therefore compared predictors of exercise tolerance in individuals requiring $>6$ minutes as well as non-participants with those who completed the LDCW within 6 minutes (table 4). In participants with OLD, quadriceps strength, IL-6 levels, and Pimax were important predictors of exercise tolerance, and the odds ratios were similar for those requiring more than 6 minutes and those who did not participate in the test. Furthermore, the odds ratios for IL- 6 and quadriceps strength were similar in participants with OLD and those with normal spirometry.

\section{DISCUSSION}

In this study OLD was associated with lower quadriceps strength and higher IL-6 and CRP levels in well functioning elderly individuals. Consistent with previous studies, we have shown an inverse association between systemic IL-6 levels and $\mathrm{FEV}_{1} \%$ predicted. Although the association appeared to be stronger in participants with OLD, the interaction $p$ value was not significant. We have also shown an important association between higher systemic IL-6 and TNF levels and lower quadriceps strength. Furthermore, the IL-6 level is an independent predictor of exercise tolerance.

The strong association between $\mathrm{FEV}_{1}$ and IL-6 in the presence of OLD is consistent with current concepts of inflammation in the pathogenesis of asthma and COPD. ${ }^{127}$ Of the three inflammatory markers studied, IL-6 had the strongest association with $\mathrm{FEV}_{1}$. The regression coefficient was higher in participants with OLD, indicating that associations between systemic IL-6 levels were stronger among participants with OLD with a similar $\mathrm{FEV}_{1}$. However, the interaction $\mathrm{p}$ value did not meet our strict criteria, and the reported differences in regression coefficients could be by chance alone. The confidence intervals for the adjusted regression coefficients for the association between $\mathrm{FEV}_{1}$ and CRP among participants with OLD compared with those with normal spirometry were similar but wider, suggesting a lack of power to detect an association in those with OLD. Although TNF is a key stimulus of IL-6 production and similar associations might be expected, ${ }^{28} 29$ the relatively short serum half life of TNF compared with IL-6 and CRP may explain the failure to find the same associations. ${ }^{30}$ This inverse association between $\mathrm{FEV}_{1}$ and systemic inflammatory markers, especially in the presence of airway obstruction, has been hypothesised to be the causal pathway for numerous systemic complications in COPD. Specifically, IL-6 which is proatherogenic, ${ }^{31}$ may explain the increased incidence of cardiovascular disease. ${ }^{32}$

Although we have previously shown an association between TNF and IL-6 with quadriceps strength in the entire Health ABC cohort, ${ }^{33}$ in this study we specifically examined the relationship in participants with OLD and those with normal spirometry. Both TNF and IL-6 levels were inversely associated with quadriceps strength in individuals with stable OLD. Levels of inflammatory markers and spirometric parameters were measured during the baseline clinic visit and participants did not report any worsening of symptoms or infections. The inverse association of IL-6 and TNF levels with quadriceps strength is consistent with our hypothesis and the results of ex vivo studies. ${ }^{8} 192034$ Again, the regression coefficients differed between participants with OLD and those with normal spirometry, but interaction $p$ values were not significant. Although participants with OLD had higher CRP levels, CRP was not an independent predictor of quadriceps strength. Compared with quadriceps strength, no association was noted between inflammatory markers and hand grip or respiratory muscle strength. Upper extremity muscle strength is preserved in patients with COPD and these results were confirmed in our study, which may explain the weak association between IL-6 and hand grip strength. Similarly, respiratory muscle strength is well preserved in patients with COPD and reduction in Pimax occurs due to hyperinflation rather than inflammatory markers. It is important to note that the quadriceps strength was approximately $10 \%$ lower in individuals with OLD than in those without OLD in our study. Previous studies in patients with clinically diagnosed COPD have reported a difference of 20$30 \%$ in quadriceps strength between COPD patients and controls. $^{35}$ The inclusion criteria for the Health ABC study included no reported difficulty with walking a quarter of a mile, climbing 10 steps, or performing activities of daily living. These selection criteria would have excluded individuals with severe OLD with the worst impairment in exercise tolerance. Furthermore, participants in the Health ABC study without OLD were 70-79 years of age. Quadriceps strength 
declines with age and this group may have lower strength than control groups in previous studies. A smaller difference in quadriceps strength was therefore noted in our study.

We also examined predictors of exercise tolerance in participants with OLD to assess the effect of inflammatory markers on exercise capacity. Individuals were subdivided into two groups based on time required to complete the LDCW test. The interval of 6 minutes was chosen to simulate the results of the 6 minute walk test. Unfortunately, $30 \%$ of the participants with OLD did not participate in the LDCW for medical reasons. These participants had the lowest quadriceps strength and higher IL-6 levels. Since excluding such a large number of participants may bias the results, we compared predictors of exercise tolerance in these participants with individuals with exercise intolerance. The similar odds ratios for these two groups suggest that the exclusion of the participants who failed to perform the LDCW test would not bias our estimates. Consistent with previous studies, quadriceps strength was a better predictor of exercise capacity than $\mathrm{FEV}_{1}$, which was not a significant predictor in the stepwise logistic regression model. ${ }^{7}$ Although IL-6 correlated with quadriceps strength, it was an independent predictor of exercise tolerance. IL-6 may be an important surrogate marker for co-morbid conditions that affect exercise capacity in this population such as cardiovascular, pulmonary, or rheumatological disease, which could explain this independent association.

Our study has limitations. Firstly, the analyses were performed using cross sectional data and therefore valid inferences regarding causal pathways cannot be drawn. Secondly, the reported associations could be explained by residual confounding, although we adjusted for known confounders that affect muscle strength and exercise tolerance. Cancer and congestive heart failure are co-morbid conditions that may affect muscle strength and inflammatory markers. Participants with cancer were excluded from the study and less than $1 \%$ of participants reported a history of congestive heart failure. Thirdly, our results cannot be generalised to other groups due to the strict inclusion criteria of the study. Individuals aged 70-79 years who could walk a quarter of a mile were included in the study. Whether individuals with severe OLD and lower quadriceps strength have a similar relationship with IL-6 is not known. Based on the linear relationship between IL-6 and $\mathrm{FEV}_{1}$ as well as quadriceps strength, one may speculate that a similar relationship would be seen in individuals with more severe OLD. Fourth, some of our participants may have asthma rather than COPD. It is often difficult to distinguish between these conditions in this population, ${ }^{36}$ and the post-bronchodilator response was not assessed. The frequency of asthma would be low in this population and asthma is not associated with raised levels of inflammatory markers. Such a misclassification bias often skews results towards the null hypothesis and is unlikely to confound the associations. Finally, our definitions of airflow obstruction and normal $\mathrm{FEV}_{1}$ were based on normative equations from population data with severity graded by ATS criteria. This is of special concern in older subjects in general, and in older minorities in particular, since the reference equations for these groups are based on a relatively small number of people. However, in the absence of any other alternative, we chose normative equations from the population data most representative of our study cohort and used the most widely accepted international criteria for defining the presence and severity of airflow obstruction. ${ }^{22}$

In conclusion, our study confirms the association between IL-6 and ventilatory limitation, reduced quadriceps strength and exercise tolerance in elderly participants with OLD as well as in those with normal spirometric parameters.
Although participants with OLD had higher inflammatory marker levels in the systemic circulation, no interaction was seen for these associations in participants with and without OLD. Systemic IL-6 levels were inversely correlated with FEV 1 and quadriceps strength and were independently associated with reduced exercise tolerance.

\section{Authors' affiliations}

S Yende, CRISMA Laboratory (Clinical Research, Investigation, and Systems Modeling of Acute Illness), Department of Critical Care Medicine, University of Pittsburgh, Pittsburgh, PA, USA

G W Waterer, Department of Medicine, University of Western Australia, Perth, Australia

E A Tolley, S Satterfield, Department of Preventive Medicine, University of Tennessee Health Science Center, Memphis, TN, USA

A B Newman, Division of Geriatric Medicine, University of Pittsburgh, PA, USA

D C Bauer, S Rubin, M Nevitt, Preventive Sciences Group, University of California, San Francisco, CA, USA

D R Taaffe, School of Human Movement Studies, University of Queensland, Australia

R Jensen, R Crapo, Pulmonary Division, LDS Hospital and University of Utah, Salt Lake City, Utah, USA

E M Simonsick, Clinical Research Branch, National Institute of Aging, Baltimore, MD, USA

T Harris, Geriatric Epidemiology Section, National Institute of Aging, Bethesda, MD, USA

S B Kritchevsky, Sticht Center on Aging, Wake Forest University School of Medicine, Winston Salem, NC, USA

This study was supported by NIA contracts N01-AG-6-2101, N01-AG6-2103, RO1-HL-074104, and N01-AG-6-2106

None of the authors has any competing interests to declare.

This work was performed while Dr Yende was at the Division of Pulmonary and Critical Care Medicine, University of Tennessee Health Science Center, Memphis, TN, USA

The study was approved by the Institutional Review Board at the University of Tennessee, Memphis and at the University of Pittsburgh, Pennsylvania.

\section{REFERENCES}

1 Gan WQ, Man SF, Senthilselvan A, et al. Association between chronic obstructive pulmonary disease and systemic inflammation: a systematic review and a meta-analysis. Thorax 2004;59:574-80.

2 Friedman GD, Klatsky AL, Siegelaub AB. Lung function and risk of myocardial infarction and sudden cardiac death. N Engl J Med 1976;294:1071-5.

3 Tockman MS, Pearson JD, Fleg JL, et al. Rapid decline in $\mathrm{FEV}_{1}$. A new risk factor for coronary heart disease mortality. Am J Respir Crit Care Med 1995; 151:390-8.

4 Curb JD, Marcus EB, Reed DM, et al. Smoking, pulmonary function, and mortality. Ann Epidemiol 1990;1:25-32.

5 Engstrom G, Lind P, Hedblad B, et al. Lung function and cardiovascular risk: relationship with inflammation-sensitive plasma proteins. Circulation 2002;106:2555-60.

6 Agnarsson U, Thorgeirsson G, Sigvaldason H, et al. Effects of leisure-time physical activity and ventilatory function on risk for stroke in men: the Reykjavik study. Ann Intern Med 1999;130:987-90.

7 Hamilton AL, Killian KJ, Summers E, et al. Muscle strength, symptom intensity, and exercise capacity in patients with cardiorespiratory disorders. Am J Respir Crit Care Med 1995; 152:2021-31

8 Debigare $\mathrm{R}$, Cote $\mathrm{CH}$, Maltais F. Peripheral muscle wasting in chronic obstructive pulmonary disease. Clinical relevance and mechanisms. Am J Respir Crit Care Med 2001;164:1712-7.

9 ATS/ERS. Skeletal muscle dysfunction in chronic obstructive pulmonary disease. A statement of the American Thoracic Society and European Respiratory Society. Am J Respir Crit Care Med 1999;159:S1-40.

10 Bernard S, LeBlanc $P$, Whittom $F$, et al. Peripheral muscle weakness in patients with chronic obstructive pulmonary disease. Am J Respir Crit Care Med 1998;158:629-34.

11 Chamberlain JS. Cachexia in cancer--zeroing in on myosin. N Engl J Med 2004;351:2124-5.

12 Acharyya S, Ladner KJ, Nelsen LL, et al. Cancer cachexia is regulated by selective targeting of skeletal muscle gene products. J Clin Invest 2004; 114:370-8.

13 Sandri M, Sandri C, Gilbert A, et al. Foxo transcription factors induce the atrophy-related ubiquitin ligase atrogin-1 and cause skeletal muscle atrophy. Cell 2004;117:399-412. 
14 Reid MB, Lannergren J, Westerblad H. Respiratory and limb muscle weakness induced by tumor necrosis factor-alpha: involvement of muscle myofilaments. Am J Respir Crit Care Med 2002;166:479-84

15 de Godoy I, Donahoe M, Calhoun WJ, et al. Elevated TNF-alpha production by peripheral blood monocytes of weight-losing COPD patients. Am J Respir Crit Care Med 1996;153:633-7.

16 Takabatake N, Nakamura H, Abe S, et al. The relationship between chronic hypoxemia and activation of the tumor necrosis factor-alpha system in patients with chronic obstructive pulmonary disease. Am J Respir Crit Care Med 2000;161:1179-84.

17 Vernooy JH, Kucukaycan M, Jacobs JA, et al. Local and systemic inflammation in patients with chronic obstructive pulmonary disease: soluble tumor necrosis factor receptors are increased in sputum. Am J Respir Crit Care Med 2002; 166:1218-24.

18 Mannino DM, Buist AS, Petty TL, et al. Lung function and mortality in the United States: data from the First National Health and Nutrition Examination Survey follow up study. Thorax 2003;58:388-93.

19 Spruit MA, Gosselink R, Troosters T, et al. Muscle force during an acute exacerbation in hospitalised patients with COPD and its relationship with CXCL8 and IGF-I. Thorax 2003;58:752-6.

20 Eid AA, lonescu AA, Nixon LS, et al. Inflammatory response and body composition in chronic obstructive pulmonary disease. Am J Respir Crit Care Med 2001; 164:1414-8.

21 Visser M, Kritchevsky SB, Goodpaster BH, et al. Leg muscle mass and composition in relation to lower extremity performance in men and women aged 70 to 79: the health, aging and body composition study. J Am Geriatr Soc 2002;50:897-904.

22 Hankinson JL, Odencrantz JR, Fedan KB. Spirometric reference values from a sample of the general US population. Am J Respir Crit Care Med 1999:159:179-87.

23 American Thoracic Society. Lung function testing: selection of reference values and interpretative strategies. Am Rev Respir Dis 1991;144:1202-18.

24 American Thoracic Society. Standardization of spirometry: 1994 update. Am J Respir Crit Care Med 1995;152:1107-36.
25 Macy EM, Hayes TE, Tracy RP. Variability in the measurement of C-reactive protein in healthy subjects: implications for reference intervals and epidemiological applications. Clin Chem 1997;43:52-8.

26 Simonsick EM, Montgomery PS, Newman AB, et al. Measuring fitness in healthy older adults: the Health $A B C$ long distance corridor walk. J Am Geriatr Soc 2001;49:1544-8.

27 Corrigan $C J$, Kay $A B$. The roles of inflammatory cells in the pathogenesis of asthma and of chronic obstructive pulmonary disease. Am Rev Respir Dis 1991;143:1165-8.

28 Yap SH, Moshage HJ, Hazenberg BP, et al. Tumor necrosis factor (TNF) inhibits interleukin (IL)- 1 and/or IL-6 stimulated synthesis of C-reactive protein (CRP) and serum amyloid $A$ (SAA) in primary cultures of human hepatocytes. Biochim Biophys Acta 1991;1091:405-8.

29 Neta R, Sayers TJ, Oppenheim JJ. Relationship of TNF to interleukins. Immunol Ser 1992;56:499-566.

30 Bazzoni F, Beutler B. The tumor necrosis factor ligand and receptor families. N Engl J Med 1996;334:1717-25.

31 Fernandez-Real JM, Vayreda M, Richart C, et al. Circulating interleukin 6 levels, blood pressure, and insulin sensitivity in apparently healthy men and women. J Clin Endocrinol Metab 2001;86:1154-9.

32 Ridker PM. Role of inflammatory biomarkers in prediction of coronary heart disease. Lancet 2001;358:946-8.

33 Visser M, Pahor M, Taaffe DR, et al. Relationship of interleukin-6 and tumor necrosis factor-alpha with muscle mass and muscle strength in elderly men and women: the Health ABC Study. J Gerontol A Biol Sci Med Sci 2002; 57:M326-32.

34 Reid MB, Li YP. Cytokines and oxidative signalling in skeletal muscle. Acta Physiol Scand 2001;171:225-32.

35 Heijdra YF, Pinto-Plata $V$, Frants R, et al. Muscle strength and exercise kinetics in COPD patients with a normal fat-free mass index are comparable to control subjects. Chest 2003;124:75-82.

36 Sciurba FC. Physiologic similarities and differences between COPD and asthma. Chest 2004;126(2 Suppl):117-124S

\section{LUNG ALERT}

Role of EGFR mutations in the pathogenesis of lung adenocarcinomas

$\Delta$ Tang $\mathrm{X}$, Shigematsu $\mathrm{H}$, Bekele BN, et al. EGFR tyrosine kinase domain mutations are detected in histologically normal respiratory epithelium in lung cancer patients. Cancer Res 2005;65:7568-72

$\mathrm{T}$ he epidermal growth factor receptor (EGFR) is found in $40-80 \%$ of non-small cell lung cancers (NSCLC). Somatic mutations of the tyrosine kinase domain of EGFR have been reported in patients with NSCLC who had clinical responses to treatment with tyrosine kinase inhibitors targeted against EGFR. However, little is known about the early pathogenesis of lung adencarcinomas and whether EGFR mutations are involved in this process.

The authors obtained surgically resected tissue specimens from 20 patients with adenocarcinomas and one with adenosquamous carcinoma, all with EGFR gene mutations. Most patients were women and never or former smokers. They also obtained tissue specimens from 16 patients with adenocarcinomas without EGFR mutation as a control group. Microdissection was done to obtain multiple foci of normal epithelium within the tumour and from adjacent normal lung tissue. DNA was extracted from cells obtained from these foci and analysed for EGFR mutation (axons 19 and 21).

Nine of the 21 patients with mutant lung adenocarcinomas $(43 \%)$ were found to have identical EGFR mutations in normal epithelium foci within the tumour. However, none of the 16 patients in the control group (without tumour EGFR mutations) had mutations in the normal respiratory epithelium. Interestingly, EGFR mutations in normal respiratory epithelium were more frequent within the tumour $(43 \%)$ than in adjacent sites $(24 \%)$.

The findings of EGFR mutations in histologically normal epithelium have not been reported before and provide a new insight into the possible mechanisms involved in initiation of peripheral airway tumours such as lung adenocarcinoma. This may provide a future target for detection or treatment. 